\title{
Design, Adjust and Reuse - How Teachers Script Pedagogical Activities
}

Ghita Jalal

Univ Lyon

INSA-Lyon

CNRS, LIRIS, UMR5205

69622, Villeurbanne, France

ghita.jalal@liris.cnrs.fr

\author{
Valentin Lachand \\ Univ Lyon \\ INSA-Lyon \\ CNRS, LIRIS, UMR5205 \\ 69622, Villeurbanne, France \\ valentin.lachand@liris.cnrs.fr
}

Aurélien Tabard
Univ Lyon
Université Lyon 1
CNRS, LIRIS, UMR5205
69622, Villeurbanne, France
aurelien.tabard@liris.cnrs.fr

Christine Michel

Univ Lyon

INSA-Lyon

CNRS, LIRIS, UMR5205

69622, Villeurbanne, France

christine.michel@liris.cnrs.fr

Permission to make digital or hard copies of part or all of this work for personal or classroom use is granted without fee provided that copies are not made or distributed for profit or commercial advantage and that copies bear this notice and the full citation on the first page. Copyrights for third-party components of this work must be honored. For all other uses, contact the owner/author(s).

Copyright held by the owner/author(s).

CHl'18 Extended Abstracts, April 21-26, 2018, Montreal, QC, Canada ACM 978-1-4503-5621-3/18/04.

https://doi.org/10.1145/3170427.3188483

\begin{abstract}
Teachers are trained to plan and conduct pedagogical activities. But, as these activities become richer -i.e. more collaborative, with more open resources, and building upon an increasing number of digital tools- planning becomes increasingly important. We conducted contextual interviews with seven middle and high school teachers, about their practices in planning and conducting pedagogical activities. We found that teachers design scripts to guide them through the session and scripts for students to use independently. They adjust their scripts during a session and edit them afterward. They reuse old scripts, adapt scripts from other teachers, and from online and physical sources. We derive implications for the design of scripting tools: supporting scripts at multiple levels of detail, or annotations for adjusting scripts during and after teaching sessions.
\end{abstract}

\section{Author Keywords}

Education; Computer Supported Collaborative Learning;

Scripting; Interview; Thematic Analysis

\section{ACM Classification Keywords}

K.3.1 [Computer Uses in Education]; H.5.2 [User Interfaces]

\section{Introduction}

Pedagogical activities involve an increasing number of interactive, collaborative and open-ended tasks. As a result, 
Components of a script We use Kobbe's framework [6] to describe script components.

Participants: The number and characteristics of the individuals that participate in a script.

Activities: A hierarchical structure of detailed instructions.

Roles: Privileges, obligations and expectations of specific participants in the group.

Resources:Physical or digital objects group members have access to teaching sessions are more dynamic and more difficult to plan. Teachers prepare these sessions ahead of time, with little knowledge of how they will unfold.

Digital teaching systems provide new ways for interacting with students. Teachers can share documents with their students, write collective blogs, online questionnaires and conduct rich collaborative activities (e.g. annotation of multimedia documents). Existing digital tools help teachers create richer, more diverse pedagogical activities for students. Yet, they provide little support for structuring or conducting these activities in class.

We need to understand why, how and for whom teachers structure - or script, in Computer Supported Collaborative Learning (CSCL) - pedagogical activities, in order to guide the design of novel interactive tools for planning and running these activities. Teachers create scripts and adjust them during and after the session, but also reuse scripts for several years, with different students, and in different contexts. Teachers need tools to interact with scripts before, during and after a teaching session.

\section{Related Work}

CSCL Scripting In collaborative learning, scripting is "the structuring of the collaboration process to make such social learning more productive" [6]. Collaborative scripts define how group members interact and collaborate to solve a problem. Dillenbourg [3] compares a collaborative script to the storyboard of a movie. The teacher creates it, and students play it, as actors would play a movie script. In collaborative scripts, or CSCL scripts, students have access to digital tools or online resources to perform collaborative tasks [12]. Well scripted, activities lead to positive results in collaborative learning, e.g., Kollar et al. found that CSCL scripts supported the acquisition of domain knowledge for all learners in a collaborative argumentation activity [7]

Script authoring tools Existing CSCL scripting tools focus on generating scripts for specific types of activities, such as collaborative argumentation, knowledge acquisition and concept mapping. These scripts are embedded in an authoring environment where teachers can edit parameters such as group size [5]. Existing script authoring tools support creating scripts for specific activities, and do not provide ways to interact with the script during and after the session. This is particularly problematic, as unexpected events occur during the session forcing teachers to adapt their original script accordingly.

Teachers design their scripts to be flexible and adaptable to classrooms events. Orchestration refers to "how teachers manage, in real time, multi-layered activities in a multiconstrained context" [9].

Orchestration tools help teachers manage the classroom environment, in real time. However, these tools rarely support "run-time scripting" [11]. Lantern and TinkerLamp [1, 4], support orchestration by increasing the teacher's awareness of how the session evolves. GroupScribble [8] embeds scripts but does not support real time script modification.

Script management tools do not support true run-time scripting. Teachers can only choose from previously scripted branches and cannot significantly deviate from, or adjust their initial script in real time. We need to better understand how teachers design and use scripts, to create novel scripting tools that support their practices.

\section{Study}

We interviewed teachers about their practices in preparing and conducting pedagogical activities. Although the use of digital tools was our primary concern, we also focused on 


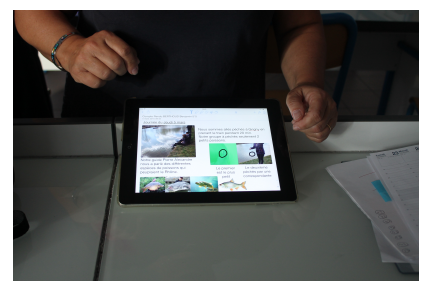

Figure 1: During the interviews, teachers showed us their scripts, how they created them, and how they used them to conduct a session.

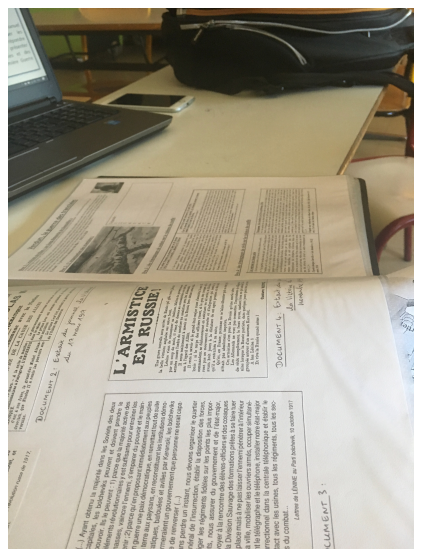

Figure 2: We interviewed participants in their classrooms. P3 created the script on paper, typed it, printed it, and used the paper version in class. existing practices using physical tools, which will inspire the design of new digital teaching tools.

Participants We interviewed seven middle and high school teachers (2 women, 5 men; age 26-50; 5 in middle school, 2 in high school) about their practices in preparing and conducting pedagogical activities. Their topics included French literature, Physics, Chemistry, History, English, German and Computer Science.

Procedure We conducted semi-structured interviews of participants in their classroom or office for about one hour. We asked participants about the tools they used to plan and conduct a recent pedagogical activity. We also asked them to show us the documents they created before, during and after the session (fig. 1). We probed for situations in which planning or running the session was particularly effective, but also when it was extremely difficult.

Data Collection We recorded audio for each interview and took written notes. We also recorded videos of participants' interactions with the documents they had created, and photographed any elements of their classroom settings (position of student tables, interactive board, tablets, routers).

Analysis We used a thematic analysis [2] approach to extract themes that describe how teachers plan and conduct pedagogical activities. We transcribed the seven interviews, and translated participants' quotes from French to English. We considered Kobbe's [6] script components (participants, activities, roles and resources). We added tools and actions, to extract individual stories of teachers interacting with physical or digital tools, to prepare and conduct a pedagogical activity. We generated initial codes from the stories. Then, we sorted the codes to create themes that describe how teachers interact with physical and digital tools, to prepare and conduct activities. Then, we reviewed the themes and mapped each story, to one or more themes.

\section{Results}

We collected 83 stories in total (between 7 and 14 stories per participant), and extracted ten themes (Table 1). We focus on practices more recurrent in participant stories. These practices appeared in stories from at least half participants, usually in multiple examples.

Not starting from scratch More than half participants $(5 / 7)$ started their scripts from samples they found online, in official textbooks or from scripts they created in previous years. P6, a German language teacher, showed us a script she will reuse: "This year, I will use the same, but I might change things depending on the students". P2, a physics teacher, showed us a folder where he keeps sample scripts from two colleagues he trusts. P7, a computer science teacher, showed us Pysequence; a python-based tool he created, along with other computer science teachers, to create scripts based on existing content. Each teacher adds sample sections, and selects, through a check-box, the sections to include in the script.

Scripting constraints before the session Almost all participants gave examples where they define constraints before the session, and specified the remaining script components (activities, resources, participants, roles) in class. For example, P1, a French literature teacher, designed an activity where students had to collaboratively write a scenario. He defined a constraint: all activities should happen within the school. Then he decided, with the students in class, on the tools, resources, and the organization of this group activity. 
Defining constraints in the script before the session

Editing the script based on students' performance

Designing interactive scripts for students

Moving between physical and digital tools

Preparing many scripts, and choosing during the session

Editing the script, according to unexpected events during the session

Not starting from scratch

Following a fixed script

Working around existing tools

Editing the script, based on students' input

Stories Participants

5

Table 1: We extracted ten themes that describe how teachers plan and conduct pedagogical activities.

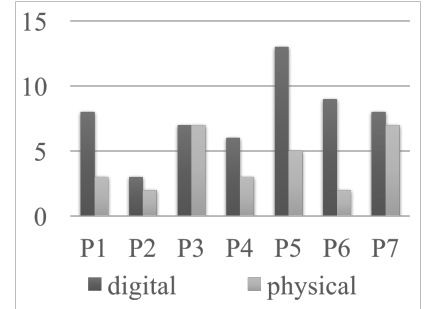

Figure 3: Teachers use digital tools more frequently in preparing and running pedagogical activities

\section{Moving between physical and digital tools Overall,} participants used digital tools more often than physical tools (paper, physical board) to design scripts (fig. 3). Four out of seven participants used general-purpose applications (like Microsoft Word), or digital teaching tools to create scripts. P6, a German language teacher, used PhotoFiltre to remove furniture from the image of a room, created separate images, and organized them in layers with workspace, a digital teaching tool compatible with her interactive board. During the session, students moved the furniture around on the interactive board, and practiced both words for furniture and prepositions of place in German.

Teachers also printed the script, or wrote it on the board. For example, P1, a French-literature teacher, explained how he takes few minutes before the start of each session to write the script on the black board: "The script I write on the board is the skeleton of my session. But again, we might do more or less than what I write on the board."

\section{Changing the script at Run-time vs after the activity} Teachers adapt their scripts during the session to the level of their students, and to live events they did not anticipate when preparing the activity. They are reflective practitioners [10]. During the session, action and reflection complement each other. Teachers assess how the activity evolves, and adjust the script accordingly.

Almost all participants (6 out of 7 ) edited the script during the session. When they used a digital tool for creating the script, and when the tool was accessible during the session, teachers edited their scripts directly on the tool. P4, a physics teacher said: "I make changes during the session, directly on the system. We finished the experiment, the questions and the exercises. In the original plan, I expected us to stop at the questions".(P4)

Otherwise, participants adjusted the script after the session for later use. Then, they integrated the changes made on paper to the digital version of the script post-session. For example, P3 a history teacher, explained how he took notes on his printed "workplan" in class. Then, in the evening, he went through his notes, and changed the script online.

Designing for the self vs Designing for students Teachers create semi-structured scripts to guide their activities.

They also create detailed scripts for students, with precise instructions to follow. Almost all participants ( 6 out of 7), created detailed scripts for sctuents. P5, an English language teacher, showed us a video she cut into several sections with EdPuzzle, a digital teaching tool. She added questions about each section: "These activities take time to prepare. But, I can squeeze them during the session, or ask students to do them at home."(P5) While the scripts teachers create for themselves can be loosely defined, those they create for students are detailed and exhaustive. 


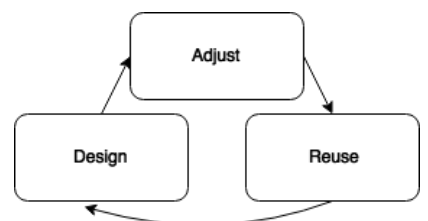

Figure 4: Teachers design scripts before the session. They adjust these scripts during the session, and integrate these changes afterwards. They reuse old scripts, and start from existing content to create their scripts.

\section{Discussion and Design Implications}

Our study revealed the importance of scripts for teachers as they prepare and run pedagogical activities. Teachers constantly interact with scripts : they create them before the session, adjust them during and after the session, and reuse them for upcoming activities (fig. 4). They use scripts as blueprints to plan, run and reflect on their sessions.

Teachers create two main types of scripts : student scripts and teacher scripts. In student scripts, teachers describe the activity in detail, and provide exhaustive instructions about running it. Teacher scripts are plans they create to help them run the activity. These scripts serve as semistructured guides for the session. They also help teachers reflect on their work, and assess their progress.

Teachers use digital tools to design, adjust, and reuse their scripts. These tools constrain teachers' interactions with their scripts. In the following, we provide design challenges for creating scripting tools that support teachers' practices as they plan and conduct pedagogical activities.

Structuring scripts at multiple levels Teachers create semi-structured scripts for themselves, and detailed scripts for students. Managing these two types of scripts requires tools that can support different levels of structuring.

Scripting tools should support creating structured, or semistructured scripts. They should support defining the components of a script (participants, resources, activities, roles) explicitly, to create activities students can run autonomously. They should also support defining some of these components, with various levels of detail. For example, teachers should be able to create scripts where they thoroughly describe the resources, but leave participants and roles open.

When they structure their scripts, teachers should be able to create and adjust links between script components. Teachers should be able to define relationships between components of their scripts, and rules for how changes in a component impact the rest of the script.

\section{Annotations for adjusting scripts during and after teach-} ing sessions Scripting tools should support quick and in situ changes to the script components (resources, participants, activity, roles). They should also support more thorough editing of the script, after the session. For example, teachers should be able to annotate their script in class. Then, they could go back to these annotations after the session.

Interacting with a script they adjusted in class might help teachers reflect on their practice. It would help them assess the activity, and adjust scripts accordingly. Teachers could annotate their scripts using text, pictures from the teaching context, or examples of students' work. For example, P3, a history teacher, explained how he used his smartphone to take pictures of a timeline the students created on the wall, using post-it notes. At home, he recreated this timeline, and used it to redesign the script of the upcoming session.

\section{Conclusion}

We conducted contextual interviews with seven teachers about their practices in planning and conducting pedagogical activities. We found that teachers design scripts that guide how they organize and run their sessions. Teachers reuse old scripts, online and physical sources to create their scripts. They adapt these scripts to unexpected events during the session, and integrate these changes after the session to prepare the script for future use. Teachers also create detailed scripts for students to use independently. These initial results reveal the many facets of interactive scripts in teachers' practices. 
Current scripting tools do not support adjusting scripts during the session, and maintaining these changes for later use. Understanding why, how and for whom teachers create scripts provides insights about the possibilities novel interactive scripting tools could offer teachers. We are building upon this work to design innovative scripting tools for teachers, supporting the design of various script types, and allowing teachers to truly manipulate scripts as they plan and conduct pedagogical activities.

\section{Acknowledgments}

This research was supported by the "FUI AAP21". We would like to thank the teachers who participated in the interviews for their valuable insights.

\section{REFERENCES}

1. Hamed S. Alavi, Pierre Dillenbourg, and Frederic Kaplan. 2009. Distributed Awareness for Class Orchestration. Springer, 211-225.

2. Virginia Braun and Victoria Clarke. 2006. Using thematic analysis in psychology. Qualitative Research in Psychology 3, 2 (Jan 2006), 77-101.

3. Pierre Dillenbourg. 2002. Over-scripting CSCL: The risks of blending collaborative learning with instructional design. Heerlen, Open Universiteit Nederland.

4. Pierre Dillenbourg. 2013. Design for Classroom Orchestration. Comput.\& Educ. 69 (Nov. 2013), 485-492.

5. Pierre Dillenbourg and Fabrice Hong. 2008. The mechanics of CSCL macro scripts. International Journal of CSCL 3, 1 (2008), 5-23.

6. Lars Kobbe, Armin Weinberger, Pierre Dillenbourg, Andreas Harrer, Raija Hamalainen, and Frank Fischer.
2007. Specifying computer-supported collaboration scripts. 2(2-3) (2007), 211 to 224.

7. Ingo Kollar, Frank Fischer, and James D. Slotta. 2007. Internal and external scripts in computer-supported collaborative inquiry learning. Learning and Instruction 17, 6 (Dec 2007), 708-721.

8. Chee-Kit Looi and Yanjie Song. 2013. Orchestration in a networked classroom: Where the teacher's real-time enactment matters. Comput.\& Educ. 69 (2013), 510 513.

9. Jeremy Roschelle, Yannis Dimitriadis, and Ulrich Hoppe. 2013. Classroom orchestration: Synthesis. Comput.\& Educ. 69 (Nov 2013), 523-526.

10. Donald A. Schon. 1984. The Reflective Practitioner: How Professionals Think In Action. Basic Books.

11. Pierre Tchounikine. 2013. Clarifying design for orchestration: orchestration and orchestrable technology, scripting and conducting. Comput.\& Educ. 69 (2013), 500-503.

12. Pierre Tchounikine. 2016. Contribution to a theory of CSCL scripts: taking into account the appropriation of scripts by learners. International Journal of CSCL 11, 3 (2016), 349-369. 